\title{
BMJ Open Quality Improving driving advice provided to cardiology patients on discharge
}

\author{
Amoolya Vusirikala, Mark Backhouse, Sarah Schimansky
}

To cite: Vusirikala A, Backhouse M, Schimansky S. Improving driving advice provided to cardiology patients on discharge.BMJ Open Quality 2018;7:e000162. doi:10.1136/ bmjoq-2017-000162

Received 11 July 2017 Revised 29 January 2018 Accepted 22 February 2018
Check for updates

North Bristol NHS Trust, Southmead Hospital, Bristol, UK

Correspondence to Dr Sarah Schimansky; sarah.schimansky@nhs.net

\begin{abstract}
Certain cardiac conditions can limit patients' ability to drive. It remains the doctors' responsibility to advise patients of any driving restrictions and is particularly important after certain diagnoses or procedures. We identified that the quality of documented advice was variable and frequently no written driving advice was recorded on discharge. It was apparent that there was a lack of awareness and knowledge of the current Driving and Vehicle Licensing Agency (DVLA) guidance among junior doctors.

We therefore designed a quality improvement project using Plan-Do-Study-Act (PDSA) methodology to improve the provision of driving advice on discharge from a cardiology ward by focusing on staff education. After collecting baseline data, we created a template with cardiologyspecific DVLA advice. During the second PDSA cycle, we improved the electronic template and also introduced a hard copy on the ward. During the third PDSA cycle, we incorporated information on DVLA guidance in the specialty induction session. We also evaluated junior doctors' confidence of providing driving advice before and after this intervention.

Baseline measurements showed that $10 \%$ (9/92) of all discharge summaries included driving advice. This improved to 49\% (34/69) after the third PDSA cycle. Importantly, after receiving information on driving advice in the induction, junior doctors felt more confident in providing driving advice to cardiology patients on discharge. In conclusion, the provision of driving advice on discharge is an important element of patient safety. However, clinicians' knowledge and awareness of current DVLA guidance is often limited. We demonstrated a significant increase in the provision of driving advice by introducing a standardised template.
\end{abstract}

\section{PROBLEM}

Southmead Hospital is a large 800-bed hospital in North Bristol. The cardiology unit consists of 24 inpatient beds, 8 coronary care unit beds and a cardiac catheter lab offering diagnostic and therapeutic interventions including percutaneous coronary intervention for patients with unstable angina and after non-ST elevation myocardial infarction (NSTEMI), pacemaker and implantable defibrillator insertion. These interventions are often associated with temporary, postprocedural driving restrictions. In addition, patients with myocardial infarctions (MIs), arrhythmias, valvular heart disease and unexplained syncope who are managed medically may also require specific driving advice. Of note, reperfusion therapy for STEMIs and cardiac surgery is not available at this site.

As junior doctors in cardiology at Southmead Hospital we noted that written guidance on driving restrictions was infrequently provided. Where available, the quality of the documented advice was variable. It was also apparent that junior doctors' knowledge of the cardiology-specific Driving and Vehicle Licensing Agency (DVLA) guidance was limited.

The lack of specific, clearly documented driving advice has serious implications for the safety of patients and the general public. Informing patients of any medical driving restrictions is a professional requirement and should form an integral part of the discharge process. Baseline measurements showed that $10 \%(9 / 92)$ of all discharge summaries included driving advice.

The primary aim was to increase the provision of appropriate written driving advice provided to patients on discharge from the cardiology ward over the course of 1 year from $10 \%$ to $100 \%$.

Secondary aims were to improve the quality of the written advice and to increase junior doctors' confidence in providing driving advice to cardiology patients in line with DVLA guidance.

\section{BACKGROUND}

Driving is a complex task and many medical conditions can affect driving ability. The DVLA publishes detailed guidance for patients and healthcare professionals on medical restrictions to driving in order to safeguard patients, passengers, pedestrians and other drivers. Group I (cars and motorcycles) and group II (heavy goods vehicles, buses and coaches) driving licences exist in the UK where group II licence holders are subject to more medical restrictions due to the size and weight of the vehicle they drive. 
With the rising burden of cardiovascular disease, the National Health Service (NHS) in 2015-2016 has seen 80000 hospital admissions primarily due to acute MI in England ${ }^{1}$ and a rise in cardiac procedures such as pacemaker insertion ${ }^{2}$ and percutaneous coronary intervention. ${ }^{3}$ These conditions and procedures can significantly limit patients' ability to drive ${ }^{4}$ and therefore have DVLA restrictions attached to them. According to current guidelines, patients who recently underwent therapeutic intervention for acute coronary syndrome or pacemaker insertion must not drive for 1 week following the procedure. ${ }^{4}$

Cardiovascular conditions, particularly syncope and arrhythmias, have been associated with increased incidence of car accidents. ${ }^{56}$ A US case-control study also found that people with heart disease and those taking cardiac medication such as ACE inhibitors were more likely to be involved in at-fault car accidents. ${ }^{7}$ These data clearly reflect the risk that patients with some cardiac conditions may pose to themselves and others if they continue to drive.

While patients are legally responsible for notifying the DVLA of any condition that affects their fitness to drive, it remains the doctor's duty to educate and inform patients of these limitations. ${ }^{48}$ However, knowledge of the DVLA guidelines among healthcare professional at all stages of their training is limited. Numerous studies have also identified a lack of knowledge about medical fitness to drive among healthcare professionals at all stages of training. ${ }^{9-12}$ For example, MacMahon et al identified that general practitioners and hospitalists who investigated patients for syncope failed to obtain a driving history in almost $90 \%$ cases. $^{9}$ As a result of inadequate or absent driving advice, many patients may continue to drive when it is medically unsafe to do so.

These findings highlight the need for educational interventions targeted at doctors and patients to increase awareness of the current DVLA guidelines. A previous study successfully improved the provision of driving advice after surgery by introducing educational measures including prewritten driving advice for discharge summaries at Southmead Hospital. ${ }^{13}$ Similarly, a group at Imperial College Healthcare NHS Trust also introduced a structured template to improve discharge advice given to patients. This intervention increased the provision of driving and flying advice from $3 \%$ to $79 \%$. $^{14}$

Based on these promising results, we decided to focus our interventions on junior doctor education and structured discharge templates to improve the quantity and quality of driving advice given to cardiology patients on discharge.

\section{MEASUREMENT}

Baseline data were collected during a predefined period from 7 December to 30 December 2015, excluding weekends and bank holidays, on the cardiology ward at Southmead Hospital. After patients were identified from the cardiology inpatient lists, their electronic discharge summaries were accessed for data collection.

A spreadsheet was used to record the date of discharge, primary diagnosis, other relevant diagnoses or comorbidities, management and presence or absence of driving advice. If written driving advice was included, we documented whether it was appropriate based on the primary diagnosis and management listed in the discharge summary.

Patients who died during admission were transferred to other wards or hospitals or did not have discharge summaries were excluded from the analysis.

Our baseline measurement from December 2015 included 108 patients for whom discharge data were unavailable in 16 cases. Also, 9 of 92 discharges (10\%) had driving advice documented. Based on either a diagnosis or intervention that warranted driving advice, $56 \%$ of discharge summaries $(n=52)$ did not include documented advice. The remaining $34 \%$ of discharge summaries $(\mathrm{n}=31)$ correctly did not include driving advice as the diagnosis did not restrict the patient's ability to drive. Notably, 19 patients were diagnosed with non-cardiac conditions.

To measure the impact of our interventions, we used the same spreadsheet to collect discharge data for cardiology inpatients during two further time periods (1-30 June 2016 and 12 December 2016 to 6 January 2017, excluding weekends and bank holidays).

Finally to assess junior doctors' knowledge of DVLA guidance, a questionnaire was sent out in December 2016 before including information on driving advice in the induction. We received five responses from junior doctors (62.5\% response rate). All five respondents indicated that they were not aware of cardiology-specific DVLA guidance and did not feel comfortable providing driving advice to cardiology patients.

\section{DESIGN}

The aim of this quality improvement project was primarily to change practice through educational interventions.

We designed the standardised driving advice template with both patients and busy junior doctors in mind. Medical terminology was avoided to facilitate patient understanding. Electronic copies were stored on all ward computers, allowing doctors to simply copy and paste the relevant driving advice into the 'advice for patient' box of the electronic discharge summary. This process was judged to be user-friendly, efficient and time-saving by other junior doctors. Initially, we had considered including a specific driving advice section to the electronic discharge summary used at this Trust. After discussion this idea was dismissed to avoid making the discharge summary too lengthy and time-consuming to complete.

To engage cardiology junior doctors, we sent out information about the project by email. They were also encouraged to express their ideas and suggestions anonymously in an online questionnaire. After discussion with 
a cardiology consultant, our driving advice template was included in the 4-monthly induction for incoming junior doctors. This intervention aimed to include senior clinicians and permanent members of the cardiology team in our project to achieve continuous staff education and a sustainable change in practice.

\section{STRATEGY}

\section{PDSA 1}

Initial baseline data were collected from review of discharge summaries in December 2015. To increase junior doctors' awareness of DVLA guidance, we created a template with standardised driving advice that was available on all ward computers and could be easily added to the electronic discharge summaries. We predicted that this intervention would make it more convenient for driving advice to be included in discharge summaries and therefore lead to an increase in documented advice.

\section{PDSA 2}

Further data were collected for discharge summaries in June 2016. To remind junior doctors to include driving advice for patients in the electronic discharge summaries, we went on to include hard copies of the template on the cardiology ward. Furthermore, we optimised the template so that it was clearly indicated the most relevant guidelines and included specific guidance for group I and group II drivers. Previously, advice for group 2 drivers had not been included in the template. These improvements were made based on feedback from junior doctors who had used the previous template. We hypothesised that these changes would sustain the change in practice seen after our first intervention and further improve the quality of written driving advice.

\section{PDSA 3}

Finally, data were collected for discharges in December 2016. The junior doctors on the cardiology ward rotate every 4 months. Therefore, we wanted to ensure that new doctors are educated about the importance of driving advice on discharge. In order to achieve continuous education and a sustainable change in practice, information on DVLA guidance and a copy of the driving advice template was included in the cardiology induction programme. A questionnaire before and after the induction helped us to assess the impact of this intervention and obtain feedback from cardiology junior doctors.

\section{RESULTS}

Our primary outcome was to increase the number of discharge summaries with documented driving advice. After introducing the first version of our template, the proportion of documented driving advice on discharge summaries increased from $10 \%(9 / 92)$ at baseline to $26 \%$ $(33 / 127)$ (figure 1).

One year after starting the project, we implemented our final intervention. By improving the template and including DVLA guidance in the cardiology specialty induction, this further increased the documented driving advice to $49 \%$ (34/69) (figures 1 and 2).

Importantly, our questionnaire revealed that none of the incoming cardiology junior doctors felt confident in providing driving advice. After the specialty induction and using our template for 2 weeks, all of the junior doctors

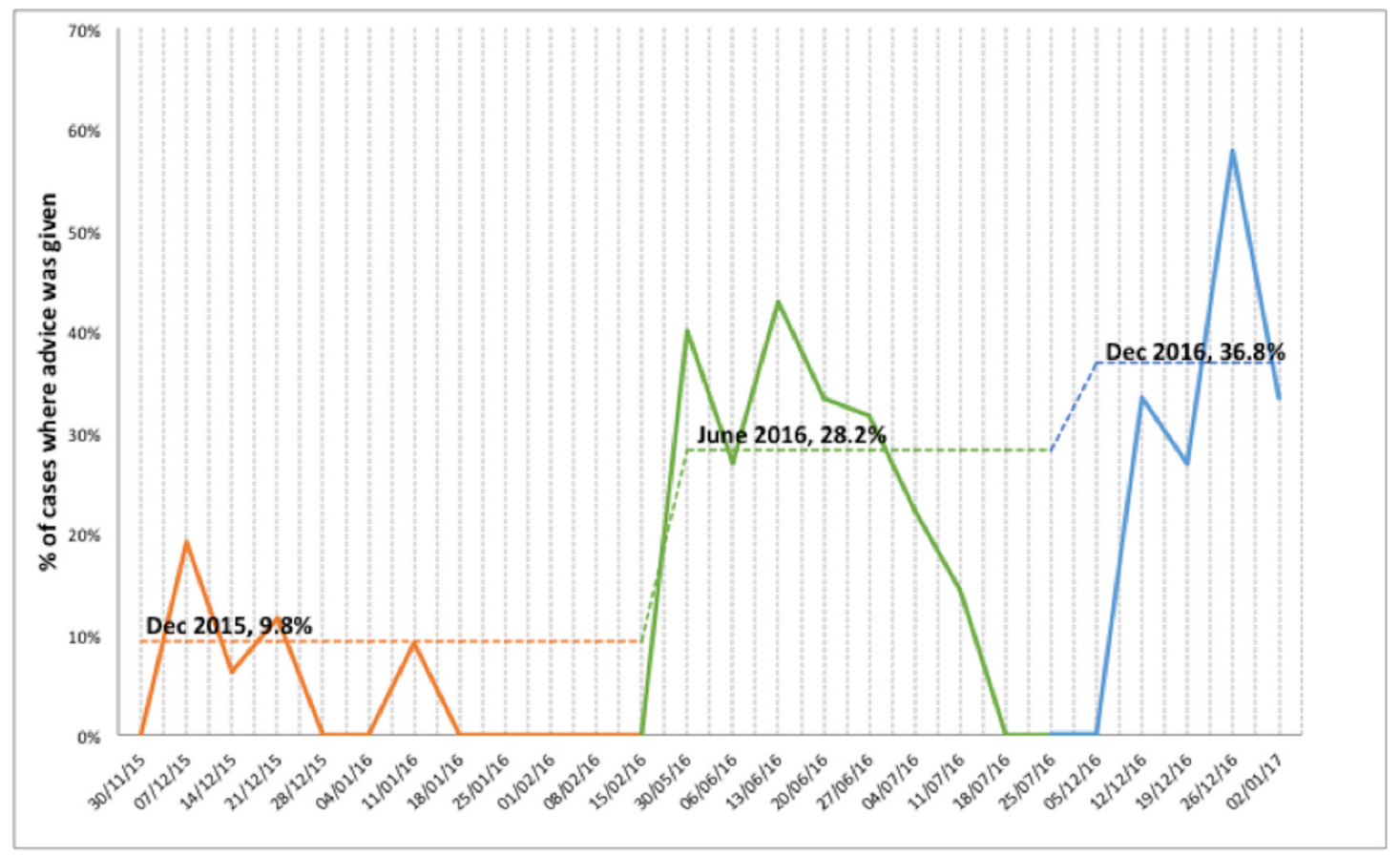

Figure 1 Statistical process U-control chart displaying sustainable increase in driving advice provision over the course of 1 year. 


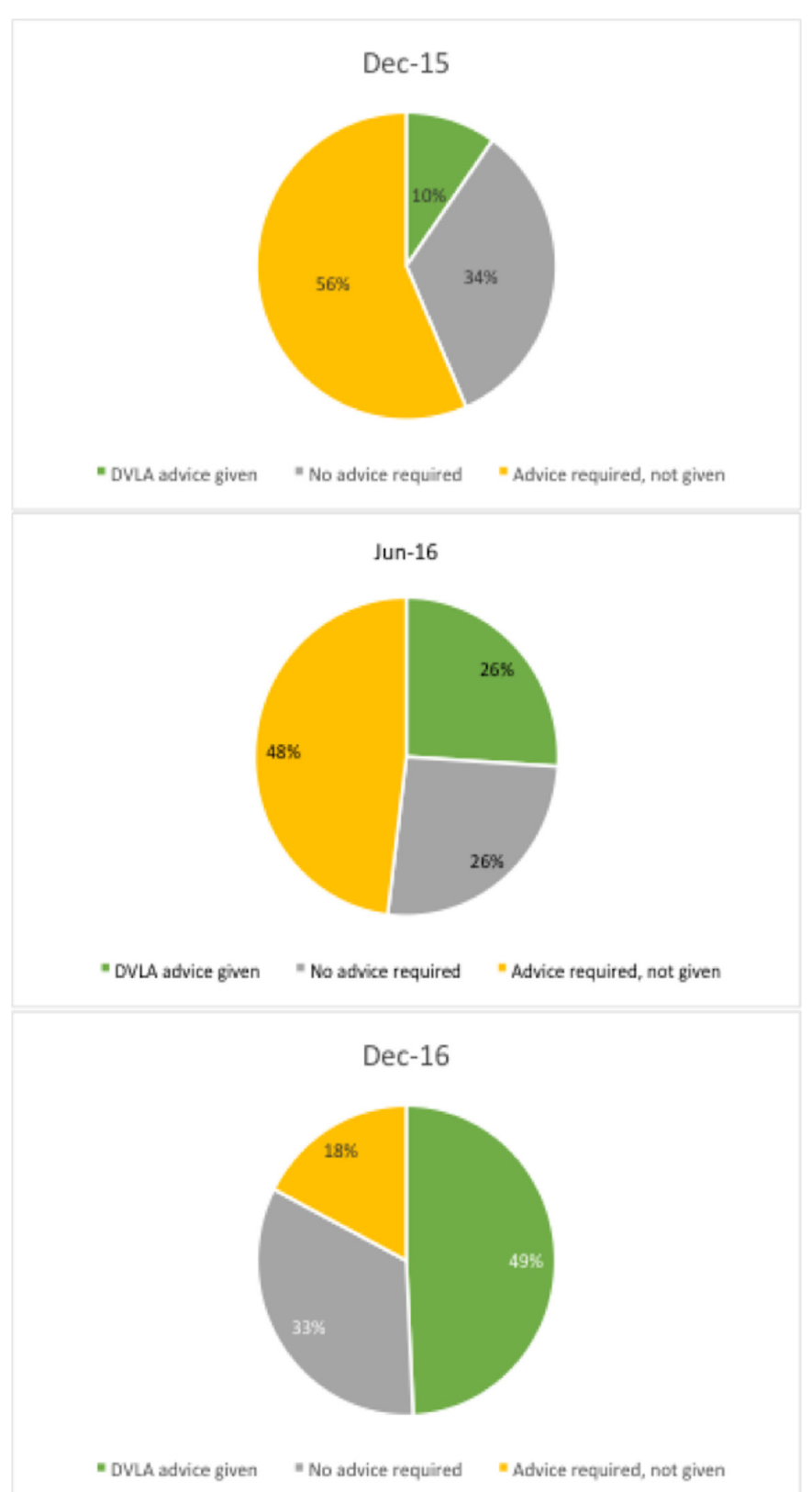

Figure 2 Pie charts displaying the number and percentage of required driving advice provided and not provided to patients admitted to the cardiology ward in December 2015, June 2016 and December 2016. DVLA, Driving and Vehicle Licensing Agency.

were aware of the DVLA guidance and felt confident in providing advice.

To ensure the data accurately reflected the impact of our interventions, we excluded weekends and bank holiday discharges from the data collection period. During weekends and bank holidays, discharge summaries are frequently written by on-call doctors who do not normally work on the cardiology ward.

Notably, the number of discharge summaries included in each cycle varied from 108 in December 2015 and 165 in June 2016 to 101 in December 2016. These numbers most likely reflect seasonal differences including fewer elective angiography procedures during the winter months.

\section{LESSONS AND LIMITATIONS}

The results demonstrate an improvement in provision of driving advice achieved through the use of simple interventions. This template and education model could be adapted for use in other departments where driving advice is frequently required. However, this is a small intervention based on one ward. Engaging busy junior doctors, who are primarily responsible for the discharges, is challenging. This small study enabled us to work closely with the doctors on the cardiology ward over the year and engaging other departments to see sustainable improvements may be more difficult. There were also issues with patients being transferred to other wards or hospitals and these were excluded from our study. However, this cohort of patients is clearly important and those responsible for their discharge may not be aware of the need to provide driving advice. Using the outcome of advice provided on discharge documentation, although convenient to measure, may be a limitation. Patients may not read the discharge summary. In addition, the absence of the driving advice in the discharge summary does not mean that the patient did not receive appropriate verbal advice from the medical team prior to discharge. It would be beneficial to record patient understanding of their driving restrictions after discharge. This would help target further interventions.

Finally, while we achieved an increase in the proportion of discharge summaries that included driving advice, just over half still lacked information on DVLA guidance.

\section{CONCLUSION}

Some cardiac conditions impair patients' ability to drive, and doctors have a responsibility to inform their patients of these restrictions. The aim of this study was to increase the provision of driving advice provided to patients on discharge from a cardiology ward. We demonstrated that simple educational interventions and a standardised template improved the quality and quantity of provided driving advice. After the interventions, the proportion of discharge summaries that included appropriate driving advice had increased from $10 \%$ to $49 \%$. Incorporating the template into junior doctor induction should ensure the sustainability of the intervention and future evaluation would help measure the long-term impact. Furthermore, it would be useful to evaluate the effect of these interventions on patients' understanding of their driving restrictions and, ultimately, the impact on road safety. Finally, providing driving advice on discharge from hospital is not limited to cardiology and similar interventions may be beneficial in other departments. Therefore, we endeavour to ensure that future foundation doctors in the hospital continue to evaluate and improve the interventions. 
Contributors All authors planned and designed this study, analysed the data and approve of the final version of this manuscript. AV collected data for December 2015, SS collected data for June 2016 and MB collected data for December 2017. The manuscript was written by all authors in equal parts and revised together. SS submitted the study on behalf of all authors.

Funding The authors have not declared a specific grant for this research from any funding agency in the public, commercial or not-for-profit sectors.

Competing interests None declared.

Provenance and peer review Not commissioned; externally peer reviewed.

Open Access This is an Open Access article distributed in accordance with the Creative Commons Attribution Non Commercial (CC BY-NC 4.0) license, which permits others to distribute, remix, adapt, build upon this work non-commercially, and license their derivative works on different terms, provided the original work is properly cited and the use is non-commercial. See: http://creativecommons.org/ licenses/by-nc/4.0/

(c) Published by the BMJ Publishing Group Limited. For permission to use (where not already granted under a licence) please go to http://www.bmj.com/company/ products-services/rights-and-licensing/

\section{REFERENCES}

1. Hospital Episode Statistics. Hospital admitted patient care activity, 2015-16. 2016 https://digital.nhs.uk/catalogue/PUB22378

2. Society BHR. National audit of cardiac rhythm management devices, 2015-16. http://www.bhrs.com/files/files/Audit Reports/CRM Devices National Audit Report 2015-16.pdf
3. BCIS. National audit of percutaneous coronary interventions, annual public report. $2015 \mathrm{http} / / / w w w . u c l . a c . u k / n i c o r / a u d i t s /$ adultpercutaneous/documents/Report-Dec2015V5.pdf

4. Driver and Vehicle Licensing Agency (DVLA). Assessing fitness to drive: a guide for medical professionals. 2016.

5. Dischinger PC, Ho SM, Kufera JA. Medical conditions and car crashes. Annu Proc Assoc Adv Automot Med 2000;44:335-48.

6. Gresset J, Meyer F. Risk of automobile accidents among elderly drivers with impairments or chronic diseases. Can J Public Health 1994;85:282-5.

7. McGwin G, Sims RV, Pulley L, et al. Relations among chronic medical conditions, medications, and automobile crashes in the elderly: a population-based case-control study. Am J Epidemiol 2000;152:424-31.

8. General Medical Council (GMC). Good medical practice. 2013.

9. MacMahon M, O'Neill D, Kenny RA. Syncope: driving advice is frequently overlooked. Postgrad Med J 1996;72:561-3.

10. King D, Benbow SJ, Barrett JA. The law and medical fitness to drive-a study of doctors' knowledge. Postgrad Med J 1992;68:624-8

11. Kelly R, Warke T, Steele I. Medical restrictions to driving: the awareness of patients and doctors. Postgrad Med J 1999;75:537-9.

12. Frampton $\mathrm{A}$. Who can drive home from the emergency department? A questionnaire based study of emergency physicians' knowledge of DVLA guidelines. Emerg Med J 2003;20:526-30.

13. Buss I, Gould L. Improving the provision of driving advice on discharge after abdominal surgery. BMJ Qual Improv Rep 2015;4:u203922.w1739.

14. Mäkelä $\mathrm{P}$, Haynes $\mathrm{C}$, Holt $\mathrm{K}$, et al. Written medical discharge communication from an acute stroke service: a project to improve content through development of a structured stroke-specific template. BMJ Qual Improv Rep 2013;2:u202037.w1095. 